\title{
Readout-Segmented Echo-Planar DWI for the Detection of Cholesteatomas: Correlation with Surgical Validation
}

\author{
(D) N. Fischer, (D) V.H. Schartinger, DD. Dejaco, DJ. Schmutzhard, H. Riechelmann, (D) M. Plaikner, and (D) B. Henninger
}

\begin{abstract}
BACKGROUND AND PURPOSE: MR imaging has become an important tool for the detection of cholesteatomas of the middle ear. Various diffusion-weighted imaging sequences are available and have shown promising results. This study aimed to evaluate readoutsegmented echo-planar DWI for the detection of cholesteatoma and compare the results with surgical validation.
\end{abstract}

MATERIALS AND METHODS: Fifty patients with chronic otitis media (24 females and 26 males; range, 12-76 years of age; mean age, 41 years) who underwent MR imaging before an operation of the middle ear (1-169 days) were included. The MR imaging protocol consisted of axial and coronal readout-segmented echo-planar DWI with b-values of 0 and $1000 \mathrm{~s} / \mathrm{mm}^{2}$ and 3-mm slice thickness. The readoutsegmented echo-planar diffusion-weighted images were fused with standard T2-weighted sequences for better anatomic assignment. The results of the MR imaging evaluation were correlated with the results from the operation.

RESULTS: Readout-segmented echo-planar DWI detected 22 of the 25 cases of surgically proved cholesteatoma. It has an accuracy of $92 \%$ ( $95 \%$ confidence interval, $80.8 \%-97.8 \%$ ), a sensitivity of $88 \%$, a specificity of $96 \%$, a positive predictive value of $96 \%$, and a negative predictive value of $89 \%$. In 1 case, a positive finding for cholesteatoma with readout-segmented echo-planar DWI could not be proved by histology, and in 3 cases, histology yielded a cholesteatoma that was not detected with MR imaging.

CONCLUSIONS: Readout-segmented echo-planar DWI is a promising and reliable MR imaging sequence for the detection and exclusion of cholesteatoma.

ABBREVIATION: RESOLVE = readout-segmented echo-planar

C holesteatoma is defined as a mass of keratinizing squamous epithelium in the tympanic cavity, mastoid cells, and the subepithelial connective tissue that can lead to an inflammatory reaction by the progressive accumulation of keratin debris and bone resorption. ${ }^{1-3}$ Cholesteatoma can only be cured by surgical removal of the entire mass. ${ }^{4}$ Depending on the surgical technique, the prevalence of residual or recurrent cholesteatoma is as high as $25 \% .^{4-7}$

The detection of cholesteatoma in patients who have undergone a middle ear operation is often difficult due to the grafts used, and regrowing squamous epithelium in the back of the middle ear or mastoid can remain symptomless for a long time. A high-resolution CT scan, which is the basic method for imaging the nonoperated middle ear, cannot reliably distinguish residual or recurrent disease

Received December 6, 2018; accepted after revision April 21, 2019

From the Departments of Otorhinolaryngology (N.F., V.H.S., D.D., J.S., H.R.) and Radiology (M.P., B.H.), Medical University of Innsbruck, Innsbruck, Austria.

Please address correspondence to Volker H. Schartinger, MD, Department of Otorhinolaryngology, Medical University of Innsbruck, Anichstraße 35, 6020 Innsbruck, Austria; e-mail: Volker.Schartinger@i-med.ac.at

http://dx.doi.org/10.3174/ajnr.A6079 from postoperative changes such as fluid, fibrous tissue, or granulations. ${ }^{8,9}$ Temporal bone CT scans have low specificity (48\%) and sensitivity (43\%) for residual or recurrent cholesteatoma. ${ }^{9}$ Thus, second-look surgery is a standard for the diagnosis of recurrent and residual cholesteatoma. However, it is associated with anesthesia and surgical risks. In approximately one-third of planned second-look procedures, a residual cholesteatoma can be found. ${ }^{10}$ In well-reconstructed middle ears with normal postoperative clinical findings and good postoperative auditory results, a second-look procedure could be avoided in two-thirds of cases.

As an alternative to second-look surgery, MR imaging has become an important tool for the detection of cholesteatoma of the middle ear. Various DWI sequences are available and have shown considerable improvement in the diagnosis of cholesteatoma, providing a variation of the conventional MR imaging sequences that use the principles of molecular diffusion or Brownian motion to generate contrast. In certain pathologic conditions, the molecular diffusion, which refers to the random movement of water molecules, is restricted. The keratin debris in cholesteatomas restricts water diffusion and produces a high signal intensity. Mu- 


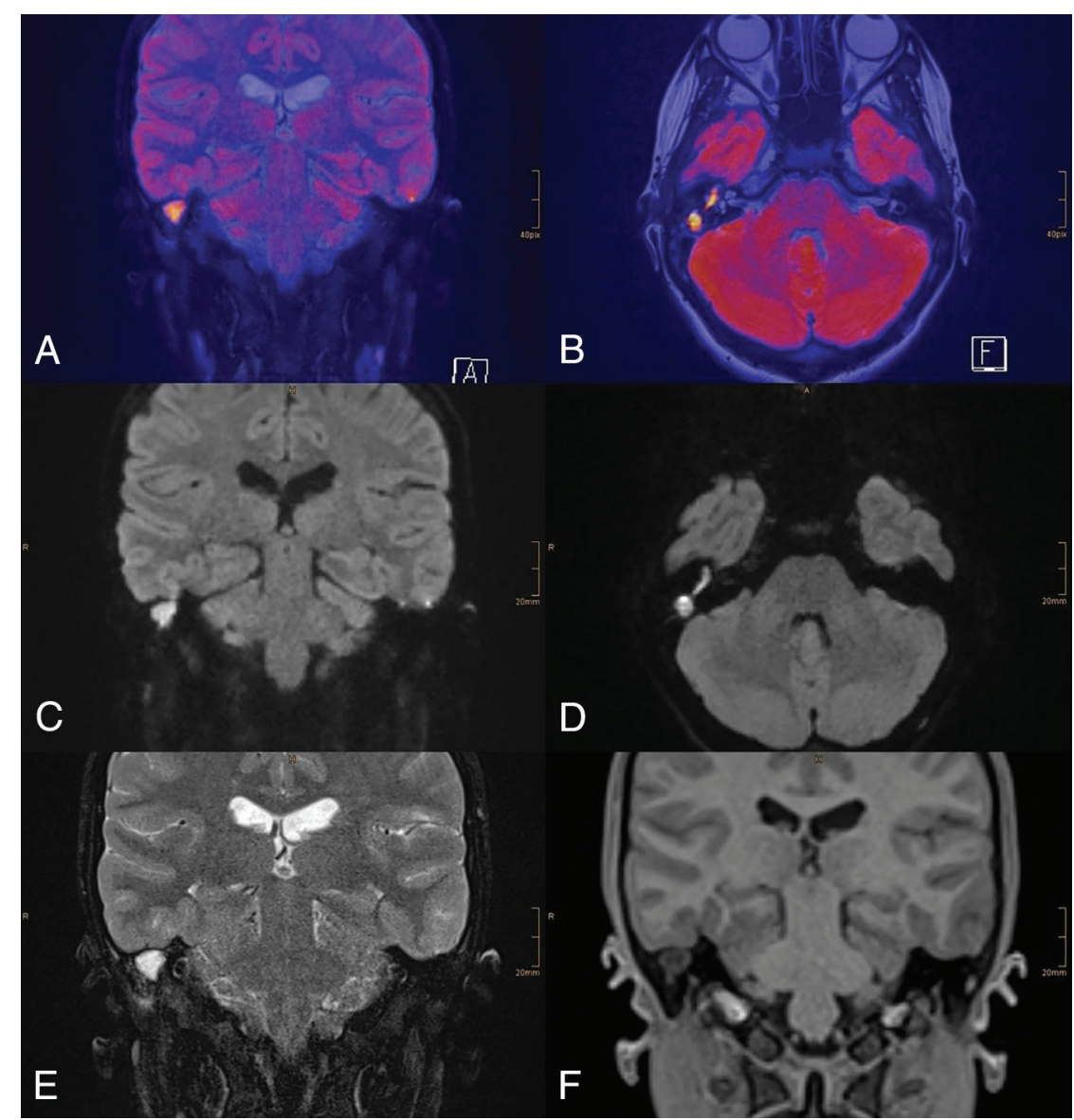

FIG 1. A 13-year-old female patient with chronic otitis media on the right side. RESOLVE-DWI shows a large hyperintense lesion on the right side. $A$ and $B$, Colored fused images of RESOLVEDWI $(b=1000)$ and T2-weighted images. $C$ and $D$, RESOLVE-DWI in coronal and axial orientations $(b=1000)$. T2-weighted image $(E)$ shows a well-delineated T2 hyperintense lesion. T1-weighted image $(F)$ shows no sign of hyperintensity. The cholesteatoma was proved intraoperatively.

cosal edema, fibrosis, and scar or granulation tissue produce a hypointense signal. DWI techniques can be divided basically into EPI-based and non-EPI-based techniques. ${ }^{11}$ Whereas EPI-DWI consists of single-shot spin-echo pulse sequences, the non-EPIDWI consists of either single-shot turbo-spin or multishot turbospin sequences. Due to different artifacts that can be generated during the acquisition of DWI, such as ghosting, motion, or susceptibility artifacts, non-EPI-DWI is recommended to avoid false-positive results. ${ }^{11,12}$

Readout-segmented echo-planar (RESOLVE)-DWI is a relatively new alternative technique for obtaining diffusion-weighted images with high quality, delivering sharp images at high spatial resolution and reduced slice thickness. RESOLVE-DWI uses the same diffusion preparation as single-shot EPI. The $k$-space trajectory is divided into multiple segments in the readout direction, so that the echo spacing is reduced compared with single-shot EPI-DWI; this feature reduces image blurring due to long echo-trains and susceptibility artifacts. Further distortion artifacts are minimized. Usually 2 spin-echoes are acquired to reduce potential phase artifacts, and the second echo is used to generate 2D navigator data for phase correction. ${ }^{11,13}$

Our purpose was to evaluate RESOLVE-DWI for the detection of cholesteatomas compared with the criterion standard intraoperative and histopathologic findings.
MATERIALS AND METHODS Study Overview

In a retrospective study at a single academic center, we analyzed patients with chronic otitis media who had undergone an operation at the Department of Otorhinolaryngology of the Medical University of Innsbruck and underwent MR imaging before the operation from November 2015 to March 2018.

A data base search was initially performed to identify all patients who had undergone a middle ear operation because of chronic otitis media. Only data of patients who underwent MR imaging before the operation were included in the study. Operative reports and histopathologic results were available for all patients. The institutional review board of the Medical University of Innsbruck approved the study (approval number: 1215/2018). Informed consent was not obtained because the data were collected retrospectively and all imaging data were pseudonymized.

\section{Imaging Technique}

MR imaging was performed with a 1.5T scanner (Magnetom Avanto-fit; Siemens Healthineers, Erlangen, Germany). The MR imaging protocol consisted of axial and coronal RESOLVEDWI with b-values of 0 and $1000 \mathrm{~s} / \mathrm{mm}^{2}$ and a $3-\mathrm{mm}$ slice thickness (19 acquired slices with each sequence). The acquisition time for each sequence was 3 minutes 1 second. In addition, T2-weighted images in coronal and axial orientations and T1weighted images with fat saturation in an axial orientation were acquired. RESOLVE-diffusion-weighted images were fused with standard T2-weighted sequences for better anatomic assignment using the software provided by the vendor. DWI was further translated into a color-coded image for better visualization.

MR images were evaluated by 2 experienced radiologists (B.H. with 12 years and M.P. with 8 years of experience in reading head and neck MR images) on the basis of standard diagnostic criteria for cholesteatoma ${ }^{11}$ with DWI. Examiners were blinded to the results of the operation and histopathology; a final decision on the presence of a cholesteatoma was made in consensus. The main diagnostic criterion for cholesteatoma on DWI is lesion hyperintensity, compared with the signal intensity of brain, on $b=0$ $\mathrm{s} / \mathrm{mm}^{2}$ images that persists or increases on high b-value (800$1000 \mathrm{~s} / \mathrm{mm}^{2}$ ) images. ${ }^{14,15}$ The so-called "T2 shine through" effect is also observed in cholesteatomas; therefore, ADC values were not integrated into our evaluation. ${ }^{11}$ Further analysis included reviewing T1-weighted images with fat saturation and T2weighted images considering known pitfalls (Fig 1). ${ }^{16}$ The colorencoded images were not included in the evaluation procedure. 


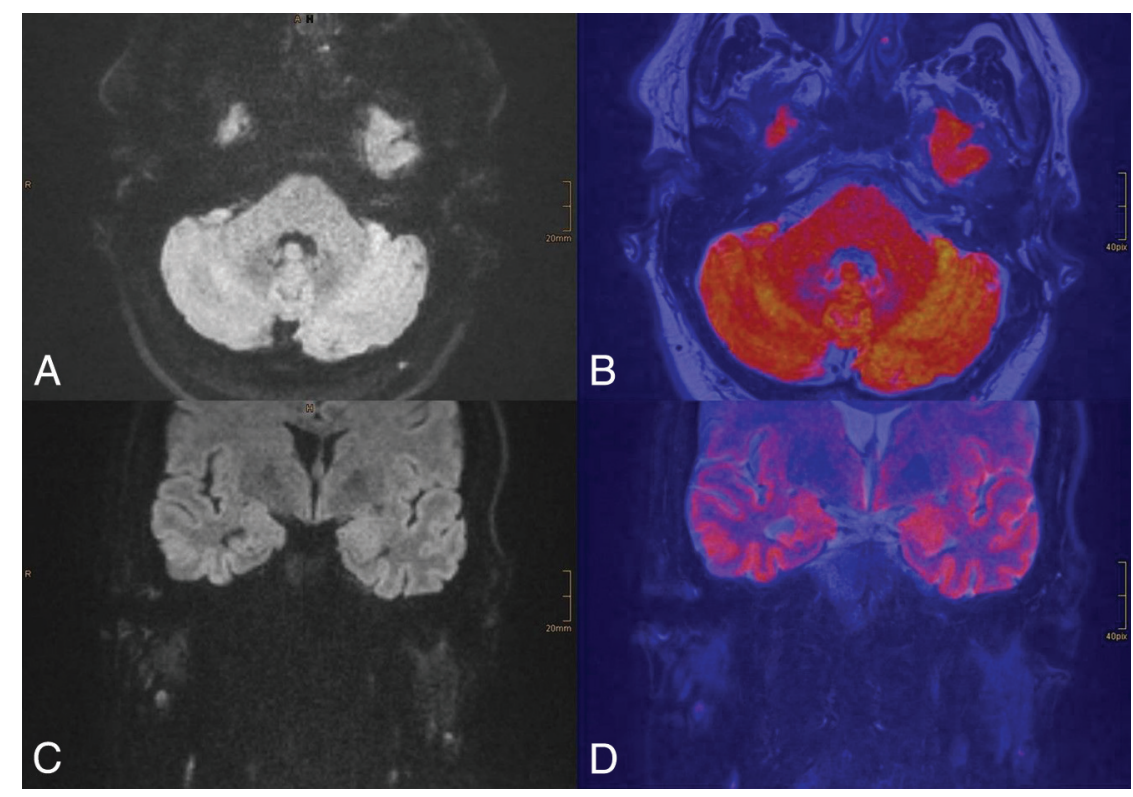

FIG 2. A 52-year-old male patient with suspected cholesteatoma on the right side. MR imaging with RESOLVE-DWI shows no sign of hyperintense signal on the right side (axial and coronal RESOLVE-DWI, $A$ and $C$, and colored fused images with T2-weighted images, $B$ and $D$ ). There were no findings on T1-weighted and T2-weighted images. Intraoperatively, a 4-mm cholesteatoma directly adherent to the malleus was detected. The small size of the lesion probably explained the false-negative results with RESOLVE-DWI.

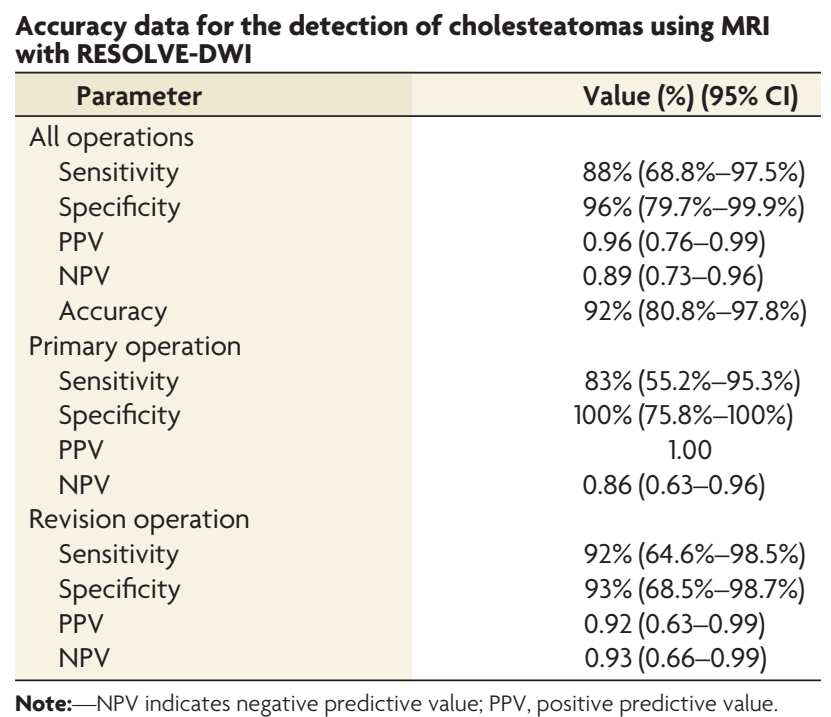

\section{Surgical Validation}

The diagnosis of cholesteatoma was made on the basis of the intraoperative presence of keratinizing squamous epithelium and debris in the middle ear and/or mastoid and pathohistologic examination of the removed tissue. Microsurgical techniques with the patient under general anesthesia were used in all patients.

\section{Data Analysis}

Numeric data were reported as mean \pm SD or mean and $95 \%$ confidence interval, and categoric data were reported as frequencies and percentages. True-positives, false-positives, true-negatives, and false-negatives were calculated from the findings on RESOLVE-DWI and surgical findings. On the basis of these data, standard diagnostic parameters were calculated. Interobserver agreement of the 2 radiologists' measurements was assessed using the Cohen $\kappa$. Statistical analysis was performed using SPSS software, Version 24 (IBM, Armonk, New York).

\section{RESULTS}

In this study, $50 \mathrm{MR}$ images (cases) of 47 patients were analyzed. Three patients underwent 2 operations. The patients were 12-76 years of age at the operation (mean age, 41 years). Twenty-four (48\%) cases were female, and 26 (52\%) cases were male. In 24 (48\%) cases, it was the first ear operation, and in 26 (52\%) cases, revision surgery was performed. The interval between imaging and the operation was 0-169 days, with a mean interval of 54 days.

The overall rate of interobserver agreement was $92 \%$ with a Cohen $\kappa$ value of $0.84 \pm 0.075$. In $25 / 50$ cases ( $50 \%$ ), a cholesteatoma was detected intraoperatively, and in $22 / 25$, there was a positive finding of surgically validated cholesteatoma on RESOLVE-DWI. In 3/25, there was no hyperintense signal on RESOLVE-DWI despite the surgical and pathohistologic proof of cholesteatoma (Fig 2). In $1 / 23$ cases, a positive finding for cholesteatoma with RESOLVE-DWI could not be proved intraoperatively. RESOLVEDWI was therefore true-positive in 22/50, true-negative in 24/50, false-positive in 1 case, and false-negative in 3/50 cases. In 46/50 cases $(92 \%)$, the radiologic and intraoperative evaluations concurred. The sensitivity for detecting cholesteatoma with RESOLVE-DWI was $88 \%$ (95\% CI, $68.8 \%-97.5 \%)$, the specificity was $96 \%$ (95\% CI, 79.7\%-99.9\%), the positive predictive value was 0.96 (95\% CI, $0.76-0.99)$, and the negative predictive value was 0.89 (95\% CI, 0.73-0.96). The diagnostic accuracy was $92 \%$ (95\% CI, 80.8\%-97.8\%).

In $12 / 24(50 \%)$ patients who had undergone an intervention on the affected ear for the first time, a cholesteatoma was detected intraoperatively. In 10/12 (83\%) cases, there was a positive finding of surgically validated cholesteatoma on RESOLVE-DWI. In the 12 surgically negative cases, the RESOLVE-DWI also showed a negative result. The sensitivity for detecting cholesteatoma with RESOLVE-DWI in unoperated ears was 83\% (95\% CI, 55.2\%$95.3 \%)$, the specificity was $100 \%$ (95\% CI, $75.8 \%-100 \%)$, the positive predictive value was 1.00 , and the negative predictive value was 0.86 (95\% CI, 0.63-0.96).

In 12/26 (46.2\%) cases with revision surgery, a cholesteatoma was diagnosed histologically, and in 11/12 (92\%) cases, there was a positive finding of surgically validated cholesteatoma on RESOLVEDWI. In 13/14 (93\%) cases in whom no cholesteatoma was found, the MR imaging also showed a negative result. The sensitivity for detecting residual cholesteatoma with RESOLVE-DWI was $92 \%$ (95\% CI, 64.6\%-98.5\%), the specificity was 93\% (95\% CI, 68.5\%98.7\%), the positive predictive value was 0.92 (95\% CI, 0.63-0.99), and the negative predictive value was 0.93 (95\% CI, 0.66-0.99). A summary of the results is shown in the Table. 


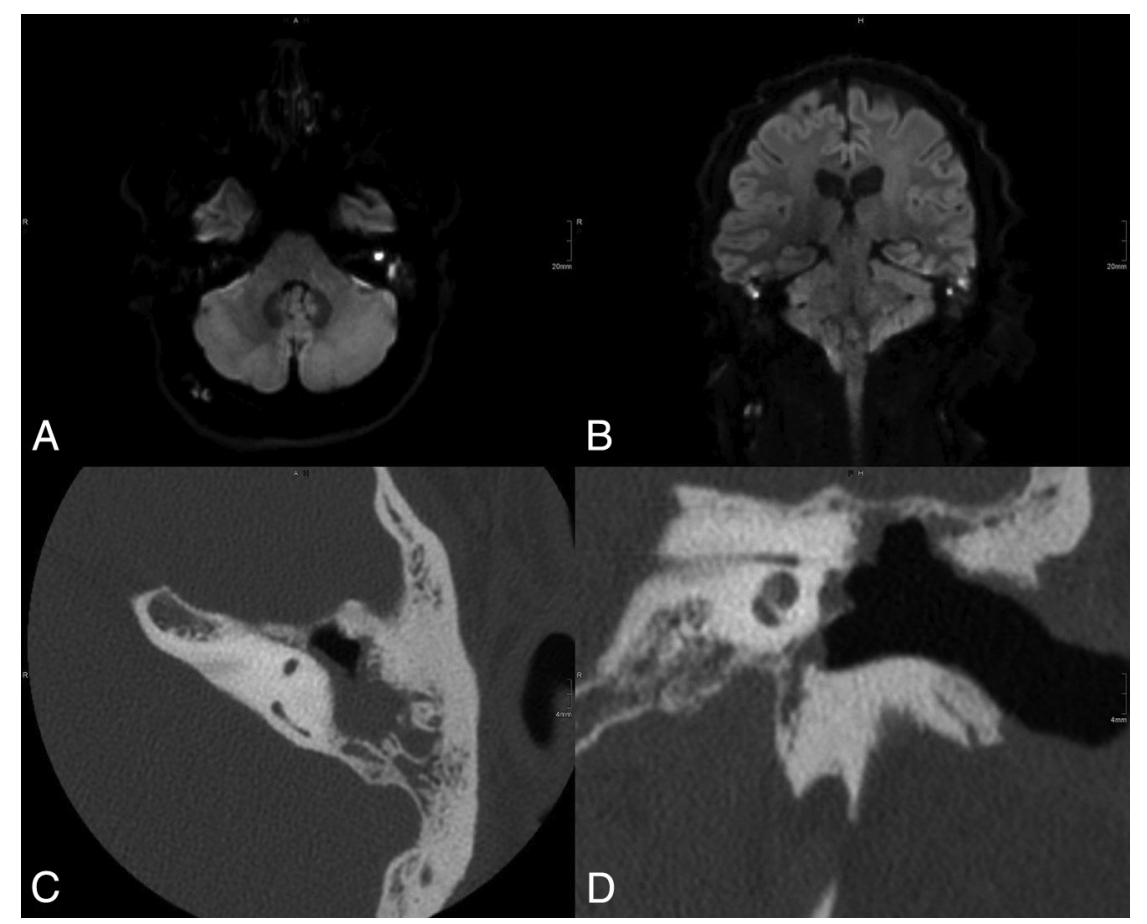

FIG 3. A 31-year-old male patient with suspected recurrent cholesteatoma on both sides. MR imaging with RESOLVE-DWI showed a hyperintense lesion on both sides highly suspicious for cholesteatoma ( $A$ and $B$ ). The patient underwent an operation of the left ear after the MR imaging. Intraoperatively, there was no sign of recurrent cholesteatoma. The reason for the false-positive MR imaging findings was probably detected wax accumulation in the open mastoid cavity. Axial (C) and coronal (D) CT scans show the radical cavity.

\section{DISCUSSION}

RESOLVE-DWI is a new technique for the diagnosis of cholesteatoma. ${ }^{11}$ So far, only a few studies have evaluated this new approach and found promising results. ${ }^{17-19}$ This study evaluated RESOLVE-DWI for the detection of cholesteatoma and compared the results with surgical validation.

Pooled sensitivity of non-EPI DWI for the detection of residual and recurrent cholesteatomas in a recent meta-analysis was $91 \%$ with a specificity of $92 \% .{ }^{20}$ In our study, RESOLVE-DWI reached a sensitivity of $88 \%$ and a specificity of $96 \%$. Therefore, in general, both sequences provide comparable results concerning the detection of cholesteatoma. In 1 patient, a positive finding for cholesteatoma with RESOLVE-DWI could not be proved intraoperatively. The reason for the high signal intensity was a wax accumulation in the open mastoid cavity (Fig 3). Lingam et al. also mentioned wax as a reason for false-positive cases: It can produce high signal changes on the $b=1000$ images and low signal and values on the ADC map. ${ }^{21}$ Thus, it is important that the open mastoid cavity be cleaned before imaging and the surgeon be aware that wax can lead to a false-positive result.

The study by Yamashita et $\mathrm{al}^{22}$ evaluated a multishot EPI sequence for the diagnosis of cholesteatoma and compared the results with a single-shot EPI sequence. They found that multishot EPI improved the accuracy of diagnosis but had no correlation with intraoperative findings or histology. Our RESOLVE sequence differs from the sequence of the Yamashita study group because it is based on a different approach using the same diffusion preparation as single-shot EPI and dividing the $k$-space trajectory into multiple segments. Potential phase artifacts are re- duced by acquiring 2 spin-echoes, with the second echo used to generate $2 \mathrm{D}$ navigator data for phase correction. Algin et $\mathrm{al}^{17}$ used a similar approach with a readout-segmented echo-planar imaging-based technique and compared this sequence with single-shot EPI. Compared with our study, the specificity was lower, at only 78\% (96\% in our study); the sensitivity was slightly higher at $100 \%$. In their study, no correlation with intraoperative findings or histology was available in patients with negative findings on MR imaging. Furthermore, they used 3T, which is, in our opinion, the worst choice for cholesteatomas due to the greater susceptibility at higher field strengths.

In 3 of 25 patients, the surgically validated cholesteatoma could not be detected with RESOLVE-DWI. The images were re-analyzed postoperatively. In 1 case, blood components (due to methemoglobin) in the middle ear, detected with T1-weighted images, caused artifacts, which led to a false-negative result because this was not considered a cholesteatoma. In the other 2 cases, the reason for the false-negative results remained unclear. We assume that these cholesteatomas could not be detected with 3-mm slice thickness RESOLVE-DWI due to their small size.

We did not encounter any relevant artifacts with RESOLVEDWI that had an influence on radiologic diagnoses in any of our cases. All recognized artifacts could be anatomically clearly assigned to the adjacent brain, which, in turn, was facilitated by the image fusion. Non-EPI-DWI is known to provide less image distortion and artifacts than other DWI techniques. ${ }^{23}$ Nevertheless, the acquisition time for non-EPI-DWI and EPI-DWI can be quite different. We found acquisition times between 3 and 6 minutes for $1 \mathrm{~b}$-value with non-EPI-DWI in the literature, ${ }^{21}$ and some studies did not indicate the acquisition time. ${ }^{12,24-26}$ Therefore, comparison between different sequences is always difficult. As with sequences used in other studies, many parameters such as slice thickness, number of slices, and FOV must be taken into account. Our RESOLVE-DWI takes 3:01 minutes for 1 orientation with 19 slices, 2 b-values, and a slice thickness of $3 \mathrm{~mm}$. In total, our protocol with 2 orientations for RESOLVE-DWI takes $<20$ minutes, including T1- and T2-weighted sequences, and delivers images with a sufficient SNR ratio. The non-EPI HASTE sequence that is provided by the manufacturer on our scanner takes much longer, with $>5$ minutes and the same slice thickness and number of images as in our RESOLVE-DWI. The acquisition would also be possible with a smaller number of slices, especially coronal slices. This could further reduce the scanning time.

The limitations of this study include a slice thickness of $3 \mathrm{~mm}$ instead of $2 \mathrm{~mm}$, which is already used in some studies. Two- 
millimeter slices are possible with the RESOLVE-DWI, but keeping the SNR at such a high level would result in a much longer scanning time. Furthermore, we performed no direct comparison with other DWI techniques such as non-EPI-DWI, but this should definitely be considered for future studies with emphasis on a correlation with histopathology. Because of the retrospective character of our study, the size of the intraoperatively found cholesteatomas was not documented and could not be further evaluated. This feature could have explained the false-negative results.

The results of this study support RESOLVE-DWI having high sensitivity and specificity for detecting cholesteatomas. Sinus tympani disease and incus erosion are associated with higher rates of cholesteatoma recurrence; thus, in these cases, a second-look operation should be considered. ${ }^{27}$ In asymptomatic patients with normal postoperative clinical findings and good hearing results, a postoperative follow-up with RESOLVE-DWI can be recommended to reduce the number of avoidable second-look procedures. $^{28}$ RESOLVE-DWI remains a promising alternative to non-EPI-DWI.

\section{CONCLUSIONS}

RESOLVE-DWI is highly sensitive and specific in identifying residual and recurrent cholesteatomas.

Disclosures: All authors declare that there are no conflict of interest related to the subject matter or material discussed in this article.

\section{REFERENCES}

1. Yung M, Tono T, Olszewska E, et al. EAONO/JOS Joint Consensus Statements on the Definitions, Classification and Staging of Middle Ear Cholesteatoma. J Int Adv Otol 2017;13:1-8 CrossRef Medline

2. Si Y, Chen YB, Chen SJ, et al. TLR4 drives the pathogenesis of acquired cholesteatoma by promoting local inflammation and bone destruction. Sci Rep 2015;5:16683 CrossRef Medline

3. Xie S, Wang X, Ren J, et al. The role of bone resorption in the etiopathogenesis of acquired middle ear cholesteatoma. Eur Arch Otorhinolaryngol 2017;274:2071-78 CrossRef Medline

4. Kuo CL, Shiao AS, Yung M, et al. Updates and knowledge gaps in cholesteatoma research. Biomed Res Int 2015;2015:854024 CrossRef Medline

5. Syms MJ, Luxford WM. Management of cholesteatoma: status of the canal wall. Laryngoscope 2003;113:443-48 CrossRef Medline

6. Westerberg J, Mäki-Torkko E, Harder H. Cholesteatoma surgery with the canal wall up technique combined with mastoid obliteration: results from primary surgery in 230 consecutive cases. Acta Otolaryngol 2018;138:452-57 CrossRef Medline

7. Haginomori S, Takamaki A, Nonaka R, et al. Residual cholesteatoma: incidence and localization in canal wall down tympanoplasty with soft-wall reconstruction. Arch Otolaryngol Head Neck Surg 2008;134:652-57 CrossRef Medline

8. Corrales CE, Blevins NH. Imaging for evaluation of cholesteatoma: current concepts and future directions. Curr Opin Otolaryngol Head Neck Surg 2013;21:461-67 CrossRef Medline

9. Tierney PA, Pracy P, Blaney SP, et al. An assessment of the value of the preoperative computed tomography scans prior to otoendoscopic 'second look' in intact canal wall mastoid surgery. Clin Otolaryngol Allied Sci 1999;24:274-76 CrossRef Medline
10. Keeler JA, Kaylie DM. Cholesteatoma: is a second stage necessary? Laryngoscope 2016;126:1499-500 CrossRef Medline

11. Henninger B, Kremser C. Diffusion weighted imaging for the detection and evaluation of cholesteatoma. World J Radiol 2017;9:217-22 CrossRef Medline

12. De Foer B, Vercruysse JP, Pilet B, et al. Single-shot, turbo spin-echo, diffusion-weighted imaging versus spin-echo-planar, diffusionweighted imaging in the detection of acquired middle ear cholesteatoma. AJNR Am J Neuroradiol 2006;27:1480-82 Medline

13. Porter DA, Heidemann RM. High resolution diffusion-weighted imaging using readout-segmented echo-planar imaging, parallel imaging and a two-dimensional navigator-based reacquisition. Magn Reson Med 2009;62:468-75 CrossRef Medline

14. Lingam RK, Connor SE, Casselman JW, et al. MRI in otology: applications in cholesteatoma and Meniere's disease. Clin Radiol 2018; 73:35-44 CrossRef Medline

15. Más-Estellés F, Mateos-Fernández M, Carrascosa-Bisquert B, et al. Contemporary non-echo-planar diffusion-weighted imaging of middle ear cholesteatomas. Radiographics 2012;32:1197-213 CrossRef Medline

16. Fukuda A, Morita S, Harada T, et al. Value of T1-weighted magnetic resonance imaging in cholesteatoma detection. Otol Neurotol 2017; 38:1440-44 CrossRef Medline

17. Algin O, Aydin H, Ozmen E, et al. Detection of cholesteatoma: highresolution DWI using RS-EPI and parallel imaging at 3 Tesla. J Neuroradiol 2017;44:388-94 CrossRef Medline

18. Azuma T, Kodama T, Yano T, et al. Optimal imaging parameters for readout-segmented EPI of the temporal bone. Magn Reson Med Sci 2015;14:145-52 CrossRef Medline

19. Holdsworth SJ, Skare S, Newbould RD, et al. Readout-segmented EPI for rapid high resolution diffusion imaging at 3 T. Eur J Radiol 2008;65:36-46 CrossRef Medline

20. Lingam RK, Bassett P. A meta-analysis on the diagnostic performance of non-echoplanar diffusion-weighted imaging in detecting middle ear cholesteatoma: 10 years on. Otol Neurotol 2017;38: 521-28 CrossRef Medline

21. Lingam RK, Nash R, Majithia A, et al. Non-echoplanar diffusion weighted imaging in the detection of post-operative middle ear cholesteatoma: navigating beyond the pitfalls to find the pearl. Insights Imaging 2016;7:669-78 CrossRef Medline

22. Yamashita K, Yoshiura T, Hiwatashi A, et al. Detection of middle ear cholesteatoma by diffusion-weighted MR imaging: multishot echoplanar imaging compared with single-shot echo-planar imaging. AJNR Am J Neuroradiol 2011;32:1915-18 CrossRef Medline

23. Castle JT. Cholesteatoma pearls: practical points and update. Head Neck Pathol 2018;12:419-29 CrossRef Medline

24. Steens S, Venderink W, Kunst D, et al. Repeated postoperative follow-up diffusion-weighted magnetic resonance imaging to detect residual or recurrent cholesteatoma. Otol Neurotol 2016;37:356-61 CrossRef Medline

25. Cimsit NC, Cimsit C, Baysal B, et al. Diffusion-weighted MR imaging in postoperative follow-up: reliability for detection of recurrent cholesteatoma. Eur J Radiol 2010;74:121-23 CrossRef Medline

26. Wong PY, Lingam RK, Pal S, et al. Monitoring progression of 12 cases of non-operated middle ear cholesteatoma with non-echoplanar diffusion weighted magnetic resonance imaging: our experience. Otol Neurotol 2016;37:1573-76 CrossRef Medline

27. McRackan TR, Abdellatif WM, Wanna GB, et al. Evaluation of second look procedures for pediatric cholesteatomas. Otolaryngol Head Neck Surg 2011;145:154-60 CrossRef Medline

28. Garrido L, Cenjor C, Montoya J, et al. Diagnostic capacity of nonecho planar diffusion-weighted MRI in the detection of primary and recurrent cholesteatoma. Acta Otorrinolaringol Esp 2015;66: 199-204 CrossRef Medline 